\title{
La conciencia pragmática en estudiantes avanzados de francés lengua extranjera: un estudio empírico ${ }^{1}$
}

\author{
Pragmatic awareness in French as a foreign \\ language advanced students: an empirical study
}

LEYRE RUIZ DE ZAROBE

Universidad del País Vasco/Euskal Herriko Unibertsitatea

leire.ruizdezarobe@ehu.eus

\begin{abstract}
Résumé
La compétence communicative a beau avoir acquis une importance croissante ces dernières années dans un monde globalisé et internationalisé, sa composante pragmatique est encore peu présente dans l'enseignement du français langue étrangère (FLE). La question se pose donc si les apprenants acquièrent une conscience pragmatique suffisante de la langue étrangère. Notre étude, fondée sur une recherche empirique, examine le niveau de conscience pragmatique d'étudiants espagnols avancés de FLE. Les résultats montrent que cette conscience est insuffisante et ne va pas de pair avec leur niveau linguistique, ce qui nous conduit à signaler le besoin de renforcer l'enseignement de la compétence pragmatique dans l'enseignement/ apprentissage du FLE.
\end{abstract}

\section{Mots-clés}

conscience pragmatique, français langue étrangère, compétence pragmatique, acquisition des langues, didactique des langues.

\begin{abstract}
The importance of pragmatic competence, which is part of communicative competence, has increased in recent years for foreign language learners in order to help them become communicative competent in a globalised and internationalised world. Nevertheless, this competence is not very present in French as a foreign language (FLE) teaching which leads us to question if learners have pragmatic awareness in the foreign language as this pragmatic awareness plays a key role in its acquisition. The aim of this study is to examine the pragmatic awareness of Spanish advanced students in FLE. Our results show that their pragmatic awareness doesnot correlate with their linguistic proficiency, which leads us to reinforce the importance of teaching pragmatic competence in FLE.
\end{abstract}

\section{Key-words}

pragmatic awareness, french as a foreign language, pragmatic competence, language acquisition, second language didactics.

1 Este artículo se enmarca en nuestro proyecto de investigación FFI2015-63715-P, financiado por el Ministerio de Economía y Competitividad (MINECO) y por el Fondo Europeo de Desarrollo Regional (FEDER). 


\section{Introducción}

La pragmática es el uso de la lengua en situación de comunicación. La pragmática es hoy objeto fundamental de aprendizaje en la didáctica de lenguas extranjeras (LE), tras un recorrido que empezó en los años 70, en la era de los métodos comunicativos, donde algunos autores hablaban de "competencia pragmática" como una de las competencias que el aprendiz de LE debe tener. Esta noción introducida en la enseñanza y aprendizaje de lenguas se remonta a Hymes (1972), quien concibe la competencia pragmática dentro de la competencia comunicativa que todo hablante debe poseer, y ha sido tratada en los años $80 \mathrm{y}$ 90 por distintos autores; los modelos más relevantes han sido los de Canale y Swain (1980), Canale (1983), Bachman (1990), Celce-Murcia et alii (1995). Todos ellos han considerado a la competencia pragmática como un componente propio en la enseñanza de lenguas extranjeras. Estos modelos de competencia comunicativa han quedado plasmados en la referencia fundamental del Marco Común Europeo de Referencia para las Lenguas (MCER, 2002). De acuerdo con el MCER (2002:13), el aprendiz de una LE ha de alcanzar una "competencia comunicativa" y "se puede considerar que la competencia comunicativa comprende varios componentes: el lingüístico, el sociolingüístico y el pragmático". En el llamado "nuevo MCER", el Volume Complémentaire avec de nouveaux descripteurs del MCER (2018: 145), se afirma que "la compétence pragmatique traite, elle, de l'utilisation réelle du langage [...] dans la (co-) construction du discours" y esta competencia se precisa en "competencia discursiva", según la cual los mensajes están estructurados y adaptados, en "competencia funcional", según la cual los mensajes realizan funciones comunicativas, y en "competencia de concepción esquemática", según la cual están organizados en esquemas interaccionales y transaccionales. Más globalmente, se considera que la competencia o habilidad pragmática en una lengua consiste en la capacidad de usar adecuadamente la lengua en un contexto social (Taguchi, 2009) o de comunicarse apropiadamente en distintos contextos socioculturales.

La competencia pragmática ha ido adquiriendo una importancia progresiva en los últimos tiempos en la enseñanza de lenguas debido a la propia evolución de nuestras sociedades, a factores como la progresiva globalización e internalización de estas, y a las nuevas necesidades que este desarrollo conlleva. Nuestras sociedades se caracterizan por ser sociedades multilingües y multiculturales, en las que personas de muy distintas lenguas y culturas necesitan interrelacionarse y comunicarse eficazmente; en este contexto, el conocimiento de la lengua no es suficiente, se requiere un conocimiento del uso sociocultural de la lengua para ser comunicativamente competente en dicha lengua, y, en ese requerimiento, la competencia pragmática resulta fundamental.

Siendo esta importancia creciente y reconocida, la realidad es que la competencia pragmática es aún poco visible en la enseñanza / aprendizaje de lenguas, en general, y del francés, en particular, y se enseña poco en los cursos de lenguas. Las razones son varias: 
primero, aunque la investigación pragmática ha avanzado considerablemente en los últimos años, se han elaborado pocos instrumentos pedagógicos para su aplicación a la enseñanza de lenguas, y esta elaboración proviene sobre todo de los investigadores en pragmática (Cohen, 2018a). Segundo, como se ve en los programas de formación de profesores, la pragmática está poco presente en estos programas, y cuando lo está, no siempre está orientada a aplicaciones prácticas (Ishihara \& Cohen, 2010), de manera que los profesores no poseen demasiada claridad sobre este componente ni tampoco sobre cómo desarrollarlo en la clase de LE. Tercero, en los métodos de lengua, tan importantes para multitud de profesores que solo disponen de este recurso, la pragmática aparece poco desarrollada, en general, en la enseñanza de cualquier LE. A este respecto, es necesario precisar que la mayor parte de la investigación existente hoy sobre la enseñanza de la pragmática, así como la elaboración de recursos pedagógicos para enseñar pragmática, se ha elaborado principalmente para el inglés, por lo que nos basaremos sobre todo en dichas investigaciones; son muy minoritarias las investigaciones sobre la enseñanza de la pragmática en francés (Ruiz de Zarobe, 2014, 2019).

Todo esto hace que profesores y alumnos no siempre posean una conciencia clara de en qué consiste enseñar o aprender pragmática. Tampoco suelen poseer una "conciencia pragmática" clara, que podemos definir como un conocimiento consciente, reflexivo y explícito sobre pragmática (Alcón y Safont, 2008), es decir, el conocimiento de las normas que subyacen al uso lingüístico adecuado en situaciones comunicativas por parte de los miembros de una determinada comunidad. En realidad, no sabemos hasta qué punto la tienen o no y, si la tienen, en qué consiste esa conciencia. Es preciso tener en cuenta que la conciencia pragmática juega un papel fundamental en la adquisición de la LE, como veremos. Por ello, dado que nuestra investigación se centra en la enseñanza/aprendizaje del FLE, estudiaremos este aspecto en la adquisición del FLE.

Este es, pues, nuestro objetivo de investigación en este artículo: examinar la conciencia pragmática de estudiantes españoles avanzados de FLE. De acuerdo con la investigación pragmática, existe una correlación entre el nivel lingüístico del aprendiz y su desarrollo pragmático (Pearson, 2018), de manera que los estudiantes más avanzados de LE serían los que tendrían una mayor competencia pragmática, y, por ende, mayor conciencia pragmática. Para llevar a cabo nuestra investigación, realizaremos una investigación empírica que explicaremos en el punto 3, tras haber analizado previamente algunos fundamentos teóricos sobre la conciencia pragmática en el aprendizaje de una LE y mostrado algunas de las investigaciones más relevantes acerca de esta cuestión.

\section{La conciencia pragmática en el aprendizaje de la LE}

Las nociones de "conciencia pragmática" y "competencia pragmática” están muy emparentadas, de manera que hoy en día se consideran prácticamente similares (Bella, 2012). 
Hemos definido la competencia pragmática como esa capacidad del aprendiz de utilizar la lengua adecuadamente en un contexto social. Una definición más precisa es la que nos proporciona Bardière (2016):

La compétence pragmatique, telle qu'elle semble se dessiner à l'issue de ces quelques considérations, pourrait se définir comme l'aptitude du locuteur/scripteur à sélectionner et à arranger au mieux les éléments linguistiques, selon la visée d'effet recherché et les circonstances énonciatives dans lesquelles il se trouve. Il s'établit donc une relation causale entre d'une part, sens d'intention/contexte situationnel et d'autre part adaptation/sélection/arrangement textuel. (Bardière, 2016: 10)

Es decir, la selección y organización de esos elementos lingüísticos que expresan la intención del hablante está determinada por el contexto situacional en el que son formulados. Esta idea se inició en autores fundamentales de pragmática, como Leech (1983) y Thomas (1983), quienes establecieron una distinción básica entre "pragmática general” y las áreas de "pragmalingüística y "sociopragmática", referidas a las condiciones de uso de la lengua. La pragmalingüística se refiere al área gramatical de la pragmática y remite a los recursos lingüísticos particulares que realizan actos comunicativos particulares (ej. rutinas pragmáticas, fórmulas de los actos de habla, etc.); la sociopragmática se refiere a los factores sociales que gobiernan el empleo de esos recursos lingüísticos en una comunidad particular. Un ejemplo: para excusarse en francés, es preciso tener acceso a las unidades pragmalingüísticas, tales como el nombre Pardon, o a la forma imperativa del verbo excuser (Excusez-moi!), o a afirmaciones que son una excusa implícita : Je regrette mon retard o a una justificación $Y$ avait des embouteillages terribles!, o a adjetivos que muestran "un état d' âme", como Désolé!, etc. Y luego es preciso evaluar el contexto de comunicación en el que se van a pronunciar: para empezar, si es apropiado o no excusarse en esa situación, después la estrategia apropiada en esa situación, si se requiere una o más estrategias (por ej. explicación o justificación), etc. Cuando no se respetan las normas pragmalingüísticas o sociopragmáticas de una lengua se producen "errores pragmáticos" (pragmatic failure, término acuñado por Thomas, 1983), que suelen tener malas consecuencias en la comunicación intercultural, tales como malentendidos, ser considerado maleducado, brusco, etc. Esta es, por cierto, una de las razones importantes para adquirir esa competencia pragmática en la LE (Ruiz de Zarobe, 2019).

Ser pragmáticamente competente significa pues poseer recursos pragmalingüísticos al mismo tiempo que recursos sociopragmáticos. Junto a esto, no solo se debe poseer esta habilidad a nivel de producción, sino igualmente a nivel de comprensión y de reconocimiento. Taguchi (2009: 2) redefine la competencia pragmática como "the ability to convey and interpret meaning appropriately in a social situation", explicitando tanto la comprensión como la producción. Ambas dimensiones son tomadas en cuenta en las definiciones más recientes de "competencia o habilidad pragmática": "Pragmatic ability is the ability to deal with meaning as communicated by a speaker (or writer) and interpreted by a listener [...] (Cohen, 2018a: 5)". 
Varios investigadores defienden que ser competente pragmáticamente presupone tener conciencia pragmática (por ej. Schmidt, 1993; García, 2004a; Alcón \& Safont 2008; Bella, 2012), es decir, un conocimiento consciente y explícito sobre pragmática, que, como hemos dicho, incluye los aspectos pragmalingüísticos y sociopragmáticos. De ahí que en los últimos años se haya defendido vehementemente la necesidad de enseñar pragmática explícitamente (cf. Taguchi 2015), con objeto de que los aprendices tengan conciencia pragmática, y, por tanto, puedan desarrollar más fácilmente esa competencia.

Distintos estudios sobre conciencia pragmática (el estudio pionero de Bardovi-Harlig \& Dörnyei, 1998; Eslami-Rakesh, 2005; Schauer, 2006; Martínez-Flor \& Alcón, 2007; Bella, 2012; Rafieyan, 2014; Takahashi, 2015) se han centrado en la capacidad del aprendiz de reconocer e identificar aspectos pragmáticos y en los efectos de la conciencia pragmática en ser competente pragmáticamente. Todos estos estudios se basan en el modelo cognitivo de adquisición de lenguas de Schmidt (1993, 2001), conocido como Noticing Hypothesis. Este modelo defiende que la información pragmática debe ser percibida conscientemente para que el aprendizaje pueda tener lugar, es decir, para que el input pueda convertirse en intake tiene que pasar por la conciencia. Este modelo alude a dos factores: noticing, como el registro de una simple ocurrencia de un evento (por ej. identificar formas lingüísticas) y understanding, como el reconocimiento de un principio o regla general (el significado de una forma lingüística en un contexto dado). A modo de ejemplo, un estudiante puede percibir que Ouvre la fenêtre! es una petición que se puede hacer a una persona muy próxima, $\mathrm{y}$, en ese caso, se utiliza el imperativo y que Tu peux ouvrir la fenêtre? puede utilizarse con otras personas menos próximas, en cuyo caso, es preciso utilizar una fórmula más indirecta y, por tanto, más cortés. La comprensión significa que el estudiante es consciente además de por qué se utiliza esta forma en el contexto del interlocutor al que se dirige. Para que se produzca un aprendizaje, $\mathrm{y}$, por tanto, una verdadera conciencia, el estudiante tiene que pasar del nivel de noticing al de understanding, por lo que se advierte la importancia de la atención y la conciencia en el aprendizaje de la pragmática.

El modelo de Smith está hoy considerado como el modelo que mejor explica el aprendizaje cognitivo de la pragmática (Takahashi, 2015; Cohen, 2018), y sugiere también cómo se puede favorecer el desarrollo de la pragmática en los aprendices. Numerosas investigaciones como las que hemos mencionado se basan en dicho modelo. Nosotros también nos basamos en este modelo para nuestra investigación.

De acuerdo con dicho modelo, ¿a qué aspectos pragmáticos debe prestarse atención para que se produzca la adquisición? ¿Locales, globales? A ambos (Ishihara, 2010b). Entonces, en coherencia con ello, examinar si nuestros estudiantes son conscientes pragmáticamente significará examinar su registro consciente de formas lingüísticas (pragmalingüística) y el reconocimiento de las formas relevantes en el contexto (sociopragmática). Esta conciencia sucede en el desarrollo tanto de las habilidades comprensivas como 
productivas: en las habilidades comprensivas, en forma de reconocimiento de elementos, y en las habilidades productivas, en forma de puesta en práctica de esos elementos pragmáticos.

Wyner (2018: 26) precisa que la habilidad pragmática implica "knowing the extent to which an utterance is acceptable and appropriate to other users of the language in conveying the speaker or writer's intended meaning". Se trata entonces de reconocer si los elementos pragmáticos de los enunciados producidos por los locutores son adecuados o no, si nos situamos en el plano de la comprensión, y de producirlos adecuadamente, si nos situamos en el plano de la producción.

Otra de las cuestiones clave determinantes de la conciencia/competencia pragmática es el nivel de lengua (LE) de los aprendices. La investigación ha mostrado que el nivel de LE afecta positivamente a la conciencia pragmática de los aprendices (Xiao, 2015), es decir, los alumnos con mayor nivel de LE poseen mayor conciencia/competencia pragmática; por ejemplo, mayor comprensión o identificación de actos de habla (Cook \& Liddicoat, 2002; García, 2004b), mayor repertorio de estrategias de realización de actos de habla (Dalmau \& Gotor, 2007), mayor capacidad de minimizar la transferencia de la L1 (Wannaruk, 2008), entre otros beneficios. Sin embargo, esta influencia positiva varía si se trata de los aspectos pragmalingüísticos o sociopragmáticos de la competencia pragmática. La investigación ha mostrado que el nivel de lengua tiene efectos positivos en la pragmalingüística de la LE, ya que los aprendices de mayor nivel poseen mayores recursos léxicos, gramaticales y discursivos (Dalmau \& Gotor, 2007; Geyer, 2007), aunque esos efectos positivos varían según los aspectos pragmáticos, como los distintos tipos de actos de habla (Cook \& Liddicoat, 2002) o la comprensión y la producción (Bardovi-Harlig, 2009). Con respecto a la sociopragmática de la LE, los aprendices de mayor nivel de lengua no parecen tener mayor conciencia de las normas sociales de la LE (Niezgoda \& Roever, 2001): "one possible reason is that L2 learners may need more time to realize differences between L1 and L2 sociopragmatic norms and make their decisions to conform to or resist the target norms" (Xiao, 2015: 572), de manera que el mayor nivel de lengua no mejora siempre la sociopragmática de la LE. En otras palabras, el nivel alto de lengua afecta más positivamente a la pragmalingüística que a la sociopragmática de la LE.

Así pues, en coherencia con la investigación realizada principalmente para el inglés, nuestras hipótesis de punto de partida son que los alumnos de nivel avanzado de FLE tendrán mayor conciencia pragmática; que esa conciencia será mayor para la pragmalingüística que para la sociopragmática; y que la conciencia pragmática habrá de tener lugar tanto en las habilidades de comprensión como de recepción.

De este modo, las preguntas de investigación que guiarán nuestra investigación son las siguientes: 
1. ¿Los estudiantes de nivel avanzado de FLE poseen conciencia sobre las normas pragmáticas de uso (pragmalingüísticas y sociopragmáticas) en la actividad de comprensión? ¿Qué tipo de conciencia?

2. ¿Los estudiantes de nivel avanzado de FLE manifiestan conciencia pragmática (pragmalingüística y sociopragmática) en sus producciones? ¿Qué tipo de conciencia?

\section{Metodología}

Exponemos a continuación la metodología que hemos llevado a cabo para realizar nuestra investigación: la identificación de los sujetos y la descripción de las pruebas.

\subsection{Los sujetos de la investigación}

Los sujetos de nuestra investigación han sido los estudiantes del último curso ( $4^{\circ}$ curso) de Grado en Filología Francesa de una universidad española. Dichos estudiantes del último curso del Grado de Filología Francesa tienen un nivel C1-C2 de francés, es decir, un nivel avanzado de francés, por lo que resultan los estudiantes idóneos para nuestra investigación, según lo expuesto en el punto anterior.

La prueba se realizó durante el mes de mayo de 2019. Los sujetos fueron 23 alumnos, 18 mujeres y 5 hombres. La lengua materna del grupo es el español.

\subsection{Las pruebas}

\subsubsection{Confección de las pruebas}

Cohen (2018c), uno de los autores principales sobre la enseñanza y evaluación de la pragmática, afirma que la competencia pragmática no es fácil de medir. Apunta algunas razones: los contextos deben estar claramente determinados, las respuestas por parte de los hablantes de una lengua sobre lo que es apropiado y no en esa lengua varía, los instrumentos de medición de la competencia pragmática resultan difíciles de crear, y otras. A pesar de ello, afirma que existen tendencias o rutinas preferidas por los nativos, que justifican la enseñanza y evaluación de la pragmática. Afirma, asimismo, la necesidad de evaluar tanto la comprensión como la producción.

Las pruebas que hemos realizado en nuestra investigación han sido dos: una de comprensión y otra de recepción. El material de las pruebas ha consistido en una selección de distintas situaciones comunicativas de la vida cotidiana que reflejan el uso auténtico de la lengua, criterio básico de las pruebas de pragmática. Hemos seleccionado distintas situaciones comunicativas extraídas de algunas guías sobre la comunicación en francés, en las que se 
proponen modelos de comunicación auténtica en francés, como, por ejemplo, la guía de Martins \& Mabilat (2016). Hemos llevado a cabo una selección de situaciones clave de la vida cotidiana, de modo que representaran situaciones sociales variadas, así como variedad de actos de habla y niveles y registros de lengua. Algunas de las situaciones presentadas pueden requerir una menor competencia pragmática, resultando a priori de mayor "facilidad" para los estudiantes que otras; sin embargo, se establece en la investigación la utilidad de mezclar situaciones diferentes, reflejo de las situaciones reales y auténticas con las que nos podemos encontrar en el día a día, para un menor condicionamiento de la prueba (Ishihara, 2010c).

En cuanto a las pruebas, exponemos a continuación las dos pruebas llevadas a cabo, una ligada a la comprensión y otra, a la producción.

\section{A. Prueba de comprensión}

El objetivo de esta prueba era estudiar si el estudiante tiene conciencia o no de las normas pragmáticas de uso de algunos enunciados lingüísticos (pragmalingüística) que hace que estos sean adecuados o no a una situación comunicativa particular (sociopragmática). La prueba consistió en presentar diez situaciones cotidianas, como las que acabamos de describir. En las situaciones aparecía subrayado algún enunciado del diálogo, o todo el diálogo, y se le formulaban dos preguntas al estudiante:

a) “¿Cómo juzgas el enunciado/el diálogo: a) adecuado b) menos adecuado c) no adecuado".

Esta pregunta está basada en una de las pruebas fundamentales que establece Ishihara (2010c) para la evaluación de la pragmática.

En pragmática no se usan los juicios "correcto" e "incorrecto", sino, como hemos visto, "adecuado" o "apropiado" o "inadecuado" o "inapropiado" cuando los enunciados no son conformes a las normas pragmáticas (Ishihara, 2010c). Una cuestión de debate en pragmática consiste en tener un criterio definido sobre la noción de "adecuado" o "no adecuado", siendo este concepto por sí mismo subjetivo. Ariel (2010:42 afirma que "pragmatic well-formedness seems less clear-cut (than grammatical), since pragmatic meaning is context bound, implicit and cancelable". Podemos afirmar que no existe un criterio fijo sobre "lo adecuado" y "no adecuado", incluso los nativos difieren sobre estos aspectos cuando se los somete a evaluar un texto. Sin embargo, podemos admitir que existen convenciones en los usos pragmáticos, usos preferidos frente a otros, que son los que nos guían con respecto a determinar la adecuación o no adecuación de los enunciados. Cohen (2010: 13) afirma a este respecto: "So, pragmatics norms refer to a range of tendencies or conventions for pragmatic language use that are not absolute or fixed but are typical or generally preferred in the L2 community". Se trata pues de tendencias y no de reglas prescriptivas o fijas.

b) “¿Por qué lo juzgas así?”

Mediante esta segunda pregunta pretendíamos tener acceso a las razones del juicio 
sobre la adecuación o no de los enunciados por parte del estudiante, lo que refleja el conocimiento metapragmático que tienen los estudiantes sobre las normas pragmáticas de uso de la L2, y con ello su conciencia pragmática. Como afirma Cohen (2018c), preguntar a los estudiantes por la razón de sus respuestas permite evaluar mejor su comprensión de la pragmática.

\section{B. Prueba de producción}

La segunda prueba es una prueba relativa a la capacidad productiva, y su objetivo es medir la adecuación pragmática del estudiante en producción, lo que nos dará indicios de su nivel de conciencia pragmática a ese nivel. La prueba consistió en presentar diez situaciones diferentes, y en pedirle al estudiante escribir lo que diría en cada una de esas situaciones si estuviera en una situación comunicativa auténtica en un país francófono. Este tipo de prueba es clásica en pragmática, se denomina "discourse-completion test" (DCT), y se ha utilizado ampliamente en la investigación (Ishihara, 2010a). En ella, el sujeto dice lo que piensa que diría en esta situación, y tiene la libertad de expresarse como quiera, ya que no existen restricciones. Aunque los DCT han sido criticados por no reflejar exactamente la espontaneidad del discurso oral, siguen siendo defendidos como pruebas eficaces de evaluación oral de la pragmática (Ishihara \& Cohen, 2010; Cohen, 2018c; Dumitrescu \& Andueza, 2018), ya que, en dichas pruebas escritas el sujeto dispone de más tiempo para reflejar su conocimiento de lo que es preciso decir en esa situación. Otra de las ventajas de los DCT, como afirma Cohen (2018c), es que resultan menos complejos de elaborar, de evaluar y de comparar que otras pruebas como los juegos de rol orales, más fidedignas de las producciones en la vida real pero, en conjunto, más dificultosas de manejar como instrumento de evaluación pragmática.

En nuestra prueba, hemos elegido diez DCT que representan diez situaciones comunes de la vida cotidiana francesa, con cinco actos de habla diferentes. Cada acto de habla aparece dos veces, pero en dos registros propios de la variedad diafásica, uno más formal y otro menos. En cada uno de ellos se proveen los factores contextuales y socioculturales que intervienen para que los estudiantes sean capaces de identificar socialmente la situación, ya que resulta fundamental que el contexto aparezca claramente delimitado.

\subsubsection{Realización de las pruebas}

Las pruebas se realizaron en el aula de clase, en el horario de una de las clases lectivas de lengua francesa. El procedimiento fue el siguiente: se anunció a los estudiantes que iban a realizar dos pruebas de francés ajenas a sus actividades universitarias, para que no se vieran condicionados. Se les explicó en qué consistían las pruebas, pero no se les dio más instrucción. La actividad empezó a las 12:00 h. de la mañana, y terminó a las 12: 45 h. Primero se repartió la primera prueba, sin límite de tiempo, que los estudiantes fueron entregando a medida que terminaban. A continuación, se repartió la segunda, también sin límite de tiempo. 


\subsubsection{Evaluación de las pruebas}

Con respecto a la evaluación de las pruebas, el criterio de evaluación fundamental ha sido el criterio mencionado de "adecuación”, tal y como hemos explicado más arriba. Ese criterio de adecuación de los enunciados o diálogos lo hemos enfocado a dos aspectos: uno, la "adecuación pragmalingüística", que, de acuerdo con Ishihara (2010c), comprende el reconocimiento o producción de las formulaciones lingüísticas de los actos de habla y el modo en que son empleadas, su corrección y riqueza lingüística; y, dos, el criterio de "adecuación sociopragmática", que comprende la adecuación de las formulaciones al contexto comunicativo de que se trate, el nivel de formalidad, la cortesía, y las normas culturales.

Nuestra evaluación de las respuestas adecuadas ha sido verificada por la evaluación añadida de dos profesores franceses nativos.

Como hemos afirmado más arriba, se prevé que los estudiantes de $4^{\circ}$ curso del Grado en Filología Francesa posean una conciencia pragmática elevada. Para medirla, nos hemos servido de la escala de evaluación de Ishihara (2010c), que distingue poor (1), fair (2), proficient (3), native-like (4). Los estudiantes habrán de alcanzar un nivel "proficient", es decir, un porcentaje en torno al 70\% en la adecuación pragmalingüística y en la adecuación sociopragmática de sus resultados en las pruebas.

\section{Análisis de los resultados}

Pasamos a analizar los resultados de ambas pruebas.

\section{A. Prueba de comprensión}

La primera prueba, en su primera parte, consistía en mostrar diez situaciones breves, y responder en cada una a la pregunta: ¿Cómo juzgas los enunciados en cursiva? con tres posibilidades de respuesta: a) adecuado b) menos adecuado d) no adecuado. Hemos contabilizado en primer lugar las respuestas apropiadas al test.

Los resultados de las respuestas apropiadas se muestran en el gráfico siguiente, donde aparecen las 10 situaciones y el porcentaje de respuestas apropiadas (sobre 23 sujetos) por situación. 


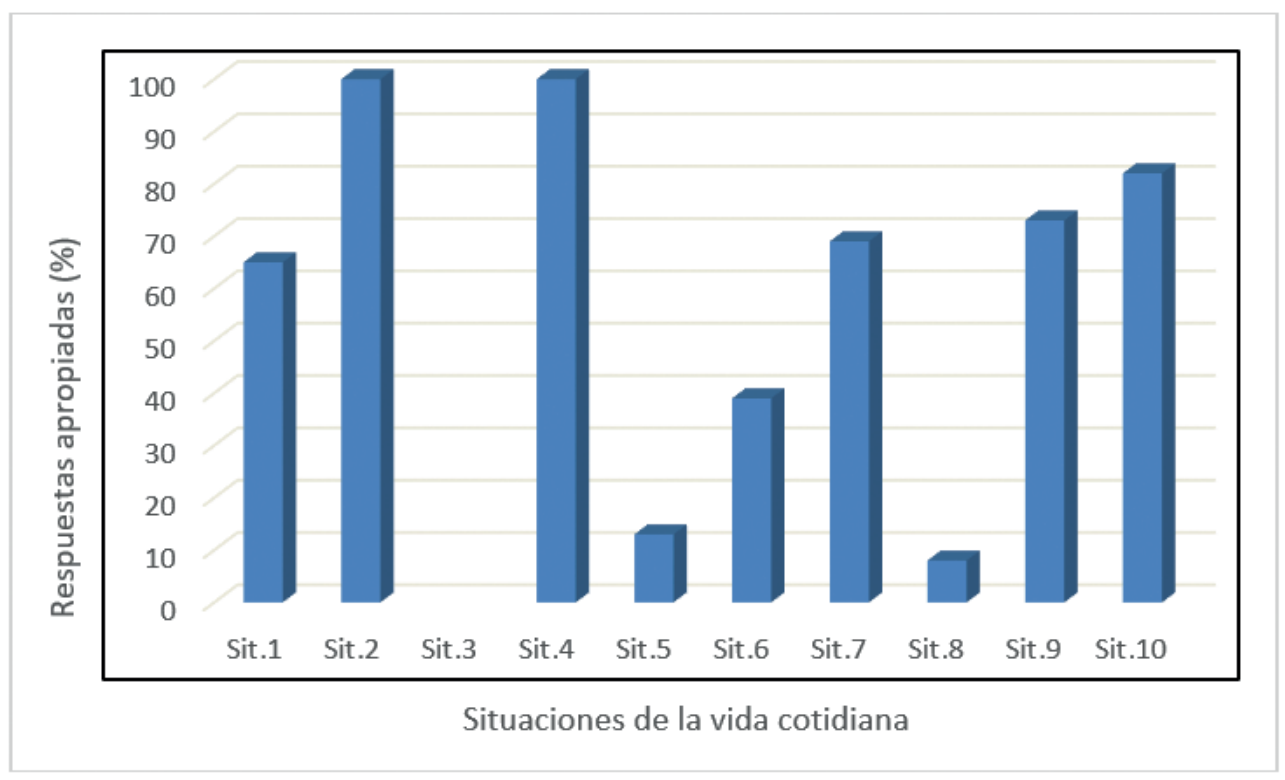

Gráfico 1. Resultados de la prueba de comprensión

Pasamos a analizar ahora las respuestas, siguiendo un orden del mayor número de respuestas apropiadas al menor.

Las respuestas apropiadas en el $100 \%$ de los casos se han producido en dos situaciones: la Sit2 y la Sit4. Esas situaciones son las siguientes:

(1) Sit2. Mme. Legrand accueille un conférencier très célèbre à l'aéroport. Le conférencier porte une valise très lourde. Mme. Legrand lui propose de l'aide. Elle dit: Je t'aide?

Se trata de un enunciado en registro familiar del acto de habla oferta. Todos los estudiantes señalan correctamente la inadecuación del enunciado del acto de habla ofrecimiento Je t'aide debido al "tutoiement" de Mme. Legrand, que se enjuicia como "manque de respect". Se supone que los hablantes no se conocen, muchos estudiantes lo señalan, y esa situación impone el "vous", afirman los estudiantes. "Il ne faut pas tutoyer les personnes inconnues. C'est la première règle française à ne pas oublier" ; "Il faut être plus polie"; 0 incluso alguien dice "Il faut parler d'une façon plus polie dans une relation professionnelle"; o "relation formelle"; o "utiliser un langage plus formel". Detectan que ese enunciado es insuficiente y que es necesario "employer certaines formes de politesse additionnelles". Como se ve, en todos aparece clara la inadecuación del "tutoiement" para esa situación entre personas desconocidas, o situación profesional. 
(2) Sit4. Yves propose à ses amis d'aller au cinéma ce soir. Il dit: "On se fait un ciné ce soir?"

La proposición de Yves es adecuada. En las valoraciones a este enunciado, los estudiantes determinan que es una conversación entre amigos y que, por lo tanto, el lenguaje es informal; "normal”; “commun"; "on peut parler librement”; "de façon plus décontractée”; "langage familier"; "langage courant"; "registre familier". Casi todos los estudiantes señalan que se trata de una conversación de amigos, y, por lo tanto, es posible expresarse así.

Con un $82 \%$ de respuestas apropiadas, figura la situación 10 :

(3) Sit10. Jean fait un compliment à Pierre sur son intelligence. Pierre répond très sérieusement: Tu sais, je ne suis pas n'importe qui.

No se trata de una respuesta habitual o frecuente a un cumplido, ya que normalmente en la cultura francesa no se ve bien elogiarse a sí mismo. El enunciado puede interpretarse irónicamente, como lo han considerado algunos estudiantes, y otros apuntan la posibilidad de considerarlo adecuado, pero si se interpreta irónicamente, lo que es correcto; en ambos casos hemos considerado las respuestas "adecuadas". "La réponse a un ton humouristique", o es "ironique" afirman. Y ese tono humorístico o irónico es lo que, para los estudiantes, justifica que se pueda emplear con amigos.

En esta situación, las explicaciones de los estudiantes que consideran el enunciado "no adecuado" son más variadas: lo tachan de "impoli"; o "malpoli"; de "manque de respect"; de respuesta "prétentieuse"; "pas humble"; "arrogante".

Con un nivel de acierto algo menor se encuentra la situación 9 y la 7:

(4) $\operatorname{Sit} 9$ (73\% de respuestas apropiadas).

Dans un vernissage.

Evelyne et Julie sont deux amies.

Evelyne: Qu'est-ce que c'est cette installation informe? Quelle horreur!

Julie: C'est le nouveau style de Jean-Yves. Tu n'aimes pas?

Evelyne: Je trouve ça nul. Dire qu'il peignait de si belles toiles, il y a quelques années!

Julie: Tu exagères. Disons que ça manque d'originalité!

El diálogo es adecuado. Las respuestas acertadas ("adecuado") lo justifican por tratarse de dos amigas en una conversación "informelle"; "familière", y por tanto, usan un "langage courant"; o "langage informel"; expresan sus emociones de una manera "tranquille et décontractée"; o "parce qu'entre amis on peut s'exprimer de cette façon". Al tratarse de una conversación entre dos amigas, no es necesario que haya "des formalités". Aunque alguien apostilla "je ne serais pas si rude, si critique", y por ello lo considera menos adecuado. Los estudiantes afirman que es la relación de amigas entre los dos personajes la que justifica la manera de expresarse en el diálogo. 
(5) Sit7 (69\% de respuestas apropiadas).

Au café:

Alexandre: Excusez-moi. C'est libre?

Une cliente: Euh...non. J'attends quelqu'un

El rechazo de la clienta puede considerarse un rechazo adecuado en esa situación. Los estudiantes, en sus aciertos, justifican el enunciado por estar en un "cadre informel"; o no muy formal; o simplemente es "correcto"; "normal". O pertenece a un "registre pas trop recherché, adéquat dans une situation quotidienne". O adecuado al contexto. O resulta útil para conseguir el objetivo de alejar a la persona.

Algunos estudiantes, aunque lo consideran adecuado, señalan que le falta cortesía. Incluso señalan las formas que le faltan como "Désolée"; "Je suis désolée". Otros, por esta misma razón, consideran el enunciado "menos adecuado", aunque creemos que este enunciado sí puede ser adecuado en una situación de una cafetería, en la que un cliente se acerca a otro y no tiene por qué requerir fórmulas de cortesía en ese caso.

(6) Sit1 (65\% de respuestas apropiadas).

À la fin d'une soirée où se sont réunis des invités, quelqu'un dit à 1'hôtesse:

- Merci beaucoup, Madame, c'était délicieux, ce plat en particulier: vous êtes un chef.

Se trata de una manera de hacer un cumplido, que podemos considerar, intensificada (Kerbrat-Orecchioni, 1992), al poseer en realidad dos cumplidos, y adecuada. La mayoría de los estudiantes la han considerado adecuada porque es "très polie", o simplemente "polie", aunque en algunos casos, a los estudiantes les parece exagerada, o un poco exagerada, y proponen suprimir la última parte. Es posible que intervengan aquí los estilos comunicativos propios, que puedan considerar demasiado efusiva esta respuesta a un elogio.

(7) Sit6 (39\% de respuestas apropiadas).

Un touriste: Excuse-moi, pour aller à la tour Eiffel, s'il te plaît?

Un passant: Vous êtes à pied?

Le touriste: Oui

Le passant: Alors, c'est très simple. Vous remontez les Champs-Elysées et au rondpoint, vous prenez l'avenue Montaigne à gauche. Ensuite, vous allez tout droit jusqu'à la place de l'Alma. Vous tournez à gauche, quai Branly. Vous marchez 300 mètres et vous arrivez aux pieds de la tour Eiffel. Vous avez compris?

Le touriste: Oui. Merci beaucoup.

Se trata de una situación que empieza cuando un turista le pregunta a alguien que pasa por la calle cómo ir a la torre Eiffel, tratándole de "tú". Se trataba de ver si los estudiantes lo identificaban y cómo lo valoraban. Para nosotros se puede considerar "menos adecuado" (en lugar de "no adecuado") ese "tutoiement", al considerar que se trata de un turista, que puede no conocer bien las reglas del francés. Los estudiantes manifiestan razones en esa lí- 
nea: "peut-être que le touriste ne connaît pas les manières du français"; "il est possible que la raison de cette erreur est que dans la langue maternelle du touriste on s'adresse aux inconnus en employant $t u$ ", como sería el caso del español. Los estudiantes han tendido a considerarlo "menos adecuado" por el "tutoiement". Las respuestas no acertadas se han producido generalmente porque no han detectado el "tutoiement" y han considerado el diálogo "adecuado" al considerarlo un diálogo "poli" o "agréable", o una forma adecuada de expresarse.

Las últimas tres situaciones corresponden a las situaciones con peores resultados (Sit5, Sit8, Sit3). Se trata de las situaciones que contenían algún error pragmalingüístico que generalmente, los estudiantes, no han detectado. Este error consiste en utilizar una forma lingüística no apropiada en esa situación, aunque sea gramaticalmente correcta.

(8) Sit5 (13\% de respuestas apropiadas).

Vous recevez une lettre qui vous donne une bonne nouvelle. La lettre commence:

Monsieur, Madame,

J'ai le bonheur de vous informer que votre candidature a été retenue.

$J^{\prime}$ ai le bonheur no corresponde a una fórmula de uso en este tipo de escrito administrativo, se trata más bien de una fórmula más propia de otros contextos más personales o familiares (nacimiento, matrimonio, etc.). La fórmula utilizada es $J^{\prime}$ ai le plaisir ... Sólo tres personas de 24 han detectado esta particularidad, y la señalan. El resto considera que es una forma adecuada de expresarse en una carta "formal"; que se trata de lenguaje formal para una carta "formelle"; y que la forma de dirigirse al destinatario es "polie", "on respecte la formalité exigée dans ce domaine", o "c'est une lettre officielle alors le langage doit être sérieux".

Algo parecido sucede en la situación siguiente:

(9) Sit8. (8\% de respuestas apropiadas)

Vous achetez un billet de train au guichet de la gare:

J'aimerais avoir un billet pour Milan

Aquí, la formula rutinaria para realizar el acto de habla de pedir un billete no es $J$ 'aimerais avoir... Aun así, esto no ha sido identificado por los estudiantes, ya que sólo 2 de 23 fueron conscientes. De los 21 restantes, la mayoría consideran que se trata de una forma "polie" de pedir un billete; o se trata de un "conditionnel de politesse"; es una forma "non violente "de dirigirse al otro; "c'est la façon normale de demander un billet"; o al contrario, varios señalan que es poco directo: "il faut être plus direct si on veut acheter quelque chose"; le falta el saludo, o el "s'il vous plaît".

No habiendo detectado el error pragmalingüístico, las opiniones se dividen entre que es cortés o no es suficientemente directo para pedir un billete.

Por último, tenemos la Sit3, con otro error pragmalingüístico, en el diálogo siguiente. 
(10) Mme. Colbert: Bonjour. Donnez-moi un rosbif d'un kilo, s'il vous plaît.

Le Boucher: Il y en a un peu plus. Ça ira quand même?

Mme. Colbert: Oui

Le Boucher: Et quoi encore?

Mme. Colbert: Deux tranches de jambon, s'il vous plaît.

La fórmula Et quoi encore? es un error pragmalingüístico, un enunciado no pragmáticamente válido en ese contexto, el correcto sería Avec ceci, fórmula estereotipada para significar qué más desea el comprador. Et quoi encore es una forma próxima a Et puis quoi encore?, que puede tener un matiz agresivo. Sin embargo, a los estudiantes les ha pasado desapercibida esa forma no adecuada, seguramente por tratarse de una transferencia del español y no ser conscientes de ella. Los estudiantes lo consideran "adecuado", encontrando el diálogo idóneo entre los dos interlocutores; o "poli”; “commun"; "courant”; "familier"; o incluso "pas trop familier"; "situation quotidienne courante". También lo justifican por la "confiance" de los interlocutores, que se encuentran recurrentemente en esa misma situación. Otros estudiantes encuentran inadecuación en la forma de hablar del carnicero, menos "polie" que la de Mme. Colbert; o la forma de hablar de Mme Colbert, "plus respectueuse" que la del carnicero, sin tener en cuenta que tanto el carnicero como la señora clienta no tienen por qué expresarse en el mismo registro, como es previsible.

Una vez analizada cada situación en particular, pasamos a analizar los resultados de forma global. Al analizar los resultados así, comprobamos que los estudiantes poseen conciencia clara del tipo de comunicación cuando dos interlocutores tienen el mismo "estatus social", en especial, cuando son amigos. Para los estudiantes, ese tipo de relación explica y justifica muchas formas de comunicación, y hace válidas expresiones que no lo serían fuera de ese contexto. Cuando dos personas son desconocidas, a veces poseen conciencia del tipo de comunicación que han de mantener, como la mujer que recoge al conferenciante del aeropuerto, y en otros no, como es el caso de la situación de la cafetería. Los resultados no son tan contundentes cuando el "estatus social" de los interlocutores es diferente, como es el caso de una señora clienta y el carnicero, o de un invitado a la fiesta y su anfitriona: en dicha situación, las opiniones de los estudiantes difieren entre sí. Situaciones estándar de la vida cotidiana francesa, donde los papeles de cada uno están bien diferenciados, como, por ejemplo, la compra en la carnicería o la compra de un billete en una estación de tren, dan asimismo lugar a valoraciones diferentes por parte de los estudiantes.

El criterio que aparece más nítido para los estudiantes en el juicio de las situaciones es el criterio de "politesse". Los estudiantes son sobre todo sensibles, de forma sobresaliente, a la cuestión de la "politesse", como uno de los indicadores principales de la adecuación de las situaciones. Es lo más repetido en todas las respuestas, como si ese indicador por sí mismo ya justificara la validez de una comunicación. Dentro del ámbito de la "politesse", aparece el tema del "tutoiement" vs. "vouvoiement", algo particular a la lengua 
francesa donde el uso del "vouvoiement" está mucho más extendido que en español; en español el trato de "usted" ha quedado reducido a muy pocos ámbitos y de mucha formalidad, en general, lo que sin duda ha influido en la falta de conciencia de los estudiantes en algún caso. A menudo los estudiantes consideran cortés un enunciado, por la forma, pero también entienden por cortés emplear fórmulas específicas de cortesía, como decir "s'il vous plaît" o "vous". Ambos criterios son válidos y pertenecen a las formas consideradas corteses en pragmática.

El otro criterio que aparece en las respuestas de los estudiantes es el de "langage/ situation formel/le"; "non formel/le"; "courant/e"; "familièr/e", es decir, criterios relativos al nivel de lengua o registro. Estos criterios son inmediatos para los estudiantes y en seguida hacen alusión a ellos.

La menor conciencia de los estudiantes se produce cuando se trata de formas lingüísticas que son correctas gramaticalmente pero que no se emplean en el contexto de uso evocado. En dos casos ha podido pasar desapercibida por la similitud de la forma en uso $J^{\prime} a i$ le bonheur frente a J'ai le plaisir; o J'aimerais avoir un billet pour Milan frente a por ej. Je voudrais acheter un billet pour Milan, lo que demandaría una competencia pragmalingüística fina de la lengua. Otras expresiones como Et quoi encore? frente a Avec ceci? han pasado desapercibidas a todos los estudiantes, probablemente por ser la primera una traducción del español.

Por último, llama la atención la búsqueda de adecuación por parte de los estudiantes en algunas situaciones. Algunas situaciones se han propuesto con la intención de resultar no adecuadas, pero el estudiante busca contextos o situaciones en las que esa comunicación sea posible, ya que, con excepción de los casos de errores pragmalingüísticos, siempre pueden imaginarse situaciones en las que un diálogo pueda tener lugar. Esta reacción de los estudiantes es normal en todo lector, que tiende a buscar pertinencia en la interpretación del sentido de un texto.

Recapitulando, y en respuesta a la primera pregunta de investigación, nuestros resultados muestran que, como capacidad comprensiva, los estudiantes poseen conciencia sociopragmática clara solo en el 50\% de las situaciones, como aquellas en la que los interlocutores se relacionan como amigos; al margen de estas situaciones, en otras determinadas por una relación entre personas desconocidas o de estatus diferente no tienen una conciencia estable. Llama la atención que algunas de esas situaciones corresponden a situaciones estándar de la vida social. El otro elemento sociopragmático en el que los estudiantes manifiestan conciencia fuerte es el de la cortesía, que suelen ligar a la formalidad, y, por tanto, a unas determinadas formas lingüísticas, aunque la atribución de dicho criterio es variable según los estudiantes, al intervenir las variaciones individuales. Son, asimismo, conscientes de los distintos registros o niveles de lengua.

Con respecto a la conciencia pragmalingüística, esta está bastante presente en la ma- 
yoría de las situaciones y es mayor que la conciencia sociopragmática. Han detectado poco los errores pragmalingüísticos en variaciones pragmáticas poco distantes de las formas adecuadas o en formas que pueden considerarse una transferencia del español. Esta conciencia pragmalingüística puede explicarse por el nivel de lengua avanzado de los estudiantes, aunque aún no discriminan totalmente las formas adecuadas de las inadecuadas.

\section{B. Prueba de producción}

La segunda prueba ha consistido en completar un cuestionario de 10 situaciones en el que se pedía a los estudiantes que escribieran lo que dirían en esa situación. Se trata de la prueba clásica en pragmática para valorar las producciones de los sujetos, denominada "discoursecompletion test", que ya hemos explicado y justificado más arriba. Para la valoración de las respuestas se ha utilizado, como para la prueba de comprensión, el criterio de "adecuación", así como las tres mismas categorías en él: 1) adecuado 2) menos adecuado 3) no adecuado.

Los resultados han sido los siguientes:

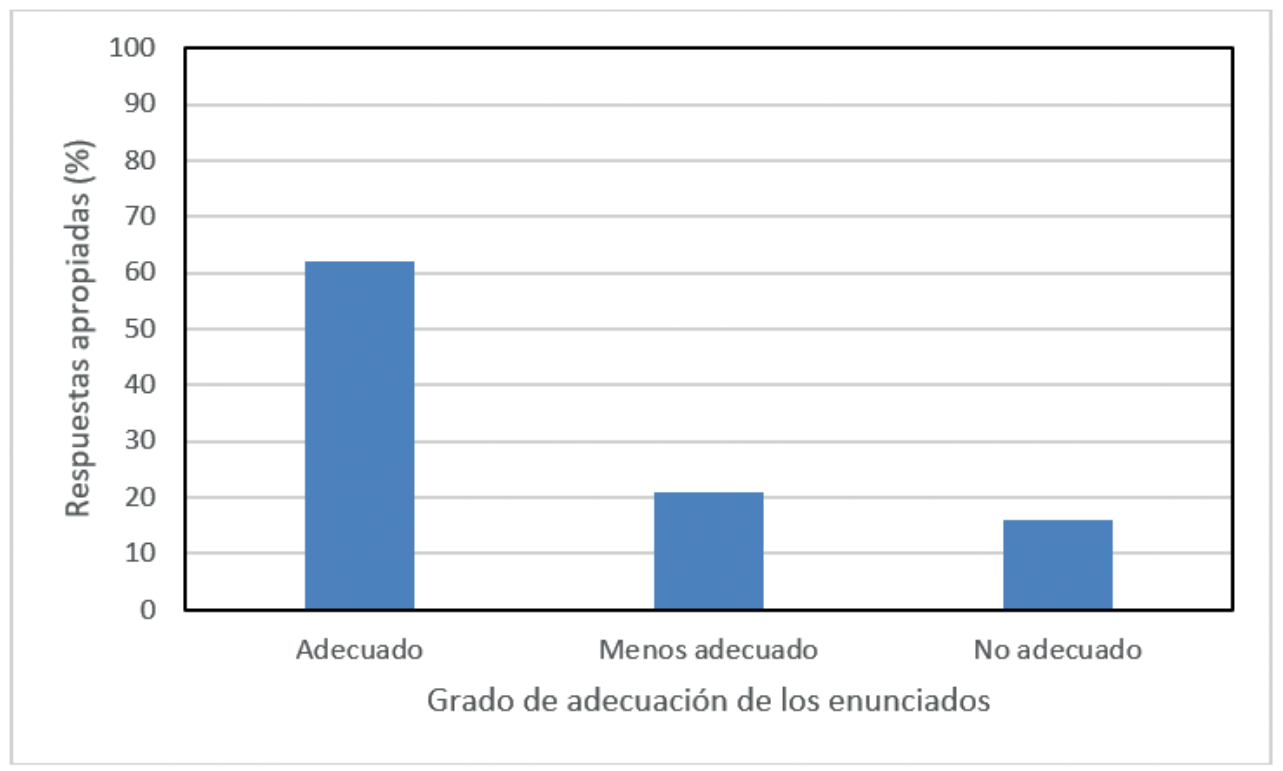

Gráfico 2. Resultados de la prueba de producción.

Pasamos a analizar los resultados. El procedimiento que seguiremos es el análisis de cada categoría de respuestas dadas por los estudiantes.

a) "Adecuado"

Un $62 \%$ de las respuestas pueden considerarse adecuadas. Veamos algunos ejemplos:

Por ejemplo, a una pregunta como: 
(1) Votre téléphone portable est tombé en panne et vous demandez à votre ami son téléphone portable pour faire un appel. Que dites vous?

Una respuesta de una estudiante como la siguiente puede considerarse válida:

Excuse-moi, mais est-ce que je pourrais t'emprunter ton portable? C'est juste une petite minute! En fait, je dois faire un appel important et je ne sais pas pourquoi, mais le mien ne marche plus.

La respuesta corresponde al acto de habla "demande". Contiene una excusa, la petición explícita, y una justificación detallada. Los dos elementos que acompañan a la propia petición, como la excusa y la justificación, se justifican porque el "tipo de imposición" de la petición es algo elevado: llamar desde el portátil del amigo. En función del "tipo de imposición" o de "amenaza" de un acto, mayor o menor, se justifica la mayor o menor "acción reparadora" que suponen esos dos elementos que acompañan a la propia petición (Brown y Levinson, 1987).

(2) Vous avez beaucoup aimé la conférence sur Proust que vous venez d'entendre. À la fin de la conférence, vous vous approchez du conférencier et vous le complimentez pour la conférence. Que dites-vous?

Mes félicitations! J'ai trouvé la conférence vraiment intéressante. J'ai beaucoup aimé.

Se trata del acto de habla "féliciter", con tres enunciados, uno nominal y dos frases, todos ellos felicitaciones. Es lo que en la terminología de Kerbrat-Orecchioni (1992) corresponde a un Face-Flattering Act ou "Acte Flatteur pour la Face", es decir, un acto positivo para el otro. Este tipo de actos tiende a ser intensificado o hiperbolizado en francés (y en muchas otras lenguas), por lo que es normal que se reitere el acto bajo la misma o distintas formas. El estilo empleado por la estudiante resulta adecuado igualmente a la persona de que se trata.

(3) Vous oubliez un rendez-vous que vous avez avec une amie pour lui expliquer un programme d'ordinateur. Une heure plus tard, vous vous en rendez compte, et vous lui téléphonez pour vous excuser. Que dites-vous?

Anne, je suis désoleé, mais j'ai complètement oublié notre rendez-vous. Si t'es encore disponible, $\mathrm{j}$ 'arrive dans 5 minutes chez toi.

Se trata de una excusa por no acudir a una cita. La interlocutora se excusa con una excusa desarrollada, no solo con la simple fórmula de excusa, y de manera adecuada. Ese desarrollo lo justifica el hecho de que no acudir a una cita no se considera una "ofensa" menor en la sociedad francesa. Así pues, la excusa contiene la propia excusa en sí, más la justificación, muy habitual en las excusas francesas, y el "offre de réparation" (Kerbrat-Orecchioni, 1992). En general, la excusa es un acto incómodo para el locutor, y demanda más o menos acción reparadora en función de la naturaleza de la "ofensa", de cómo lo considere el que la ha cometido, etc. 
(4) Vous aimez beaucoup la robe rouge que porte votre copine d'appartement. Vous lui faites un compliment. Que dites-vous?

Oh la la, comme tu es belle aujourd'hui! Cette robe est superbe, avec elle, tu as l'air super canon, crois-moi.

Se trata de una respuesta a un cumplido, que en realidad se compone de tres cumplidos. Como hemos indicado antes, los "actos de habla halagadores de la imagen" tienden a ser intensificados en francés, con objeto de reforzar el cumplido.

(5) Une bonne copine à vous, Anne, vous demande de l'aide avec le travail de littérature qu'elle doit présenter en classe. Vous voudriez bien l'aider, mais vous avez un examen demain et vous refusez. Que dites-vous?

Désolée, Anne, je voudrais bien t'aider, mais j'ai un examen demain. De toutes façons, si tu as des questions, tu peux me téléphoner, ok?

En esta producción una estudiante realiza de manera apropiada el siempre delicado acto de rechazar una petición, compuesta por el rechazo, la justificación, y una oferta de solución.

b) "Menos adecuado".

Un $21 \%$ de respuestas han sido "menos adecuadas". En esta categoría ha aparecido un tipo de producciones que comparten un denominador común: las producciones contienen menos información pragmática que la que darían los nativos, resultan más escuetas o más directas. Esto puede deberse a la limitación de la prueba, dado que los sujetos no se encuentran realmente viviendo una situación auténtica, y quizás se limitan a decir lo esencial. También puede deberse a que los aprendices de LE, en general, tienden más a emplear actos directos que indirectos (Blum-Kulka et alii, 1989; Holtinnen, 2017). Y también, en algún caso, puede deberse a la influencia del español, cuyo estilo puede considerarse quizás un poco más directo que el francés.

Ejemplos de estas respuestas son:

(6) Vous montez dans le bus et vous bousculez une dame âgée qui est sur le point de tomber par terre. Que dites-vous?

Excusez-moi, Madame.

En una situación real es casi seguro que, tratándose de que ha empujado a una señora mayor que por poco se cae en el autobús, el locutor no se limitaría a esa fórmula de excusa, añadiría otros procedimientos discursivos para excusarse, como reduplicar las excusas, decir, por ejemplo, que no se ha dado cuenta, que ha sido sin querer, preguntarle si se encuentra bien, etc. 
(7) Vous oubliez un rendez-vous que vous avez avec une amie pour lui expliquer un programme d'ordinateur. Une heure plus tard, vous vous en rendez compte, et vous lui téléphonez pour vous excuser. Que dites-vous?

Excuse-moi Marie j'ai complètement oublié notre rendez-vous.

En dicha excusa, este enunciado parecería insuficiente para "reparar" el acto de habla y esperaríamos algún componente más de los habituales en las excusas, que compensen la molestia del acto.

En estos dos últimos casos, el enunciado producido por el/la estudiante resulta limitado, ya que cada acto de habla, en su actualización comunicativa, se compone de uno o más enunciados, que son los que se producen en el discurso real.

Otro tipo de producción que hemos considerado en esta categoría "menos adecuado" son casos como el siguiente:

(8) Une bonne copine à vous, Anne, vous demande de l'aide avec le travail de littérature qu'elle doit présenter en classe. Vous voudriez bien l'aider, mais vous avez un examen demain et vous refusez. Que dites-vous?

Pas aujourd'hui, Anne! J'ai la tête dans mes affaires.

En principio, este rechazo se consideraría rudo. Es evidente que toda respuesta es posible, máxime en función de la relación entre los interlocutores, pero no es la respuesta más adecuada.

\section{c) "No adecuado"}

Las producciones no adecuadas (16\%) son aquellas que no siguen las normas pragmalingüísticas ni sociopragmáticas en francés, que no corresponden al estilo comunicativo francés ni al apropiado a una situación. Mostramos algunos ejemplos:

(9) Vous aimez beaucoup la robe rouge que porte votre copine d'appartement. Vous lui faites un compliment. Que dites-vous?

On pourrait se changer la robe un jour. J'aimerais porter cette robe rouge que tu portes maintenant.

(10) Vous invitez votre chef de bureau à votre anniversaire chez vous. Que dites-vous? Vous avez été très bon avec moi et je serais très honoré de vous tenir chez moi pour mon anniversaire.

O esta otra respuesta:

(11) Monsieur X, je m'adresse à vous pour vous inviter à mon anniversaire. Votre assistance est très importante pour moi. 
Otras producciones no adecuadas son aquellas que resultan no corteses, cortantes o rudas en francés, como el rechazo a cuidar del perro:

(12) J'ai pas de temps pour votre chien, j'suis très occupée.

O el rechazo a ayudar a la compañera de clase con el trabajo de literatura, con una respuesta sorprendente:

(13) Est-ce que tu m'as aidé quand j'en avais besoin? Où étais-tu alors?

Si analizamos los resultados de la prueba 2 de forma global, un $62 \%$ de las respuestas de los estudiantes en producción son adecuadas desde el punto de vista pragmalingüístico y sociopragmático, es decir, los estudiantes poseen conciencia pragmática en el $62 \%$ de los casos. La adecuación sociopragmática tiene en cuenta los distintos contextos comunicativos para emitir los enunciados que resultan apropiados a ellos. La adecuación pragmalingüística en esta prueba está relacionada con la realización lingüística de los actos de habla, principalmente, que es adecuada tanto en la formulación lingüística del enunciado principal que lo realiza como en el número de enunciados que se emplean, así como en el tipo de enunciados que acompañan a ese enunciado principal. Asimismo, en ese porcentaje de casos los estudiantes tienen conciencia de la cortesía, ya que esa cantidad de enunciados que realizan un acto de habla son fruto de la acción reparadora que supone el acto, y se consideran "procedimientos de cortesía", en sentido pragmático (Brown y Levinson, 1987).

Los casos de menor adecuación, el $21 \%$, corresponden sobre todo a casos de menor adecuación sociopragmática y están relacionados precisamente con la construcción de actos de habla más completos y comunicativos, como se lleva a cabo en situación real. No sabemos si los resultados de la prueba han estado condicionados por el tipo de prueba o no, o son fruto de la falta de conciencia del estudiante. También corresponden a casos de falta de cortesía, pero no de manera tan extrema como en los casos no adecuados.

En un $16 \%$ de casos, los estudiantes producen enunciaciones inadecuadas, que no corresponden al estilo comunicativo francés o no son aptas para usarse en la situación social dada.

En general, nos ha llamado la atención que en las respuestas apenas se han cometido errores lingüísticos que hayan podido interferir en el resultado.

Recapitulando, y en contestación a la segunda pregunta de investigación, los estudiantes manifiestan conciencia pragmalingüística y sociopragmática en producción en un $62 \%$ de casos, cifra no tan elevada como esperaríamos. Esto quiere decir que el nivel lingüístico no presupone el nivel pragmático, al contrario de lo que señalan algunas investigaciones previas, como hemos mostrado en el punto 2. En el resto de casos en que manifiestan menor conciencia pragmática $(21 \%)$, esta menor conciencia es sobre todo sociopragmática está 
relacionada con la realización de actos de habla de manera más similar a la de los nativos en situación auténtica, o en la ausencia de la cortesía (pragmática) que requieren determinados actos de habla. Siendo sensibles, como lo son estos estudiantes, a la cortesía en general, no lo son siempre al nivel de cortesía demandado por determinados actos de habla en determinadas situaciones en la sociedad francesa, generalmente más cortés que la española (Kerbrat-Orecchioni, 2005). La falta de conciencia pragmática se produce en no tan pocos casos (16\%).

Para terminar, nos gustaría añadir que, tanto en producción como en comprensión, las dos pruebas de pragmática han estado condicionadas por algo que sucede siempre en pragmática, y es que no existe una única forma de ser pragmáticamente adecuado, como hemos mencionado más arriba, incluso para los nativos. La subjetividad es un factor que interviene decisivamente en pragmática, como hemos visto en las pruebas, y como comprobamos en la vida corriente, como locutores en nuestra propia lengua materna.

\section{Conclusión}

La competencia pragmática se reconoce hoy en día como una competencia fundamental en el aprendizaje de una LE, como el francés, si bien se trata de una competencia con poca presencia en el aula de lengua, particularmente en FLE. Nuestro estudio ha mostrado que nuestros estudiantes de mayor nivel formativo poseen bastante conciencia pragmática pero no alcanzan el nivel "proficient" y dicha conciencia no está correlacionada con su nivel lingüístico, es decir, se esperaría que en su nivel lingüístico dicha conciencia fuera más elevada. Su conciencia pragmática tiene las características siguientes: la conciencia pragmalingüística es mejor en comprensión que en producción, donde aparecen errores pragmalingüísticos; la conciencia sociopragmática es clara para algunas situaciones de la vida cotidiana, como las interacciones entre amigos, y menos clara para interacciones donde los interlocutores pertenecen a un "estatus social" diferente, incluso en situaciones estándar. Destaca la alta conciencia de la cortesía de los estudiantes (vouvoiement, formalidad, nivel de lengua...), en general, y menos, la conciencia relativa a la cantidad de cortesía (cortesía pragmática) que conllevan determinados actos de habla. Podemos decir que, en general, los estudiantes tienen mayor conciencia pragmalingüística que sociopragmática, la primera más ligada a lo lingüístico y la segunda, más a lo social, en coherencia con los resultados de la investigación realizada sobre todo para el inglés. Esto podría deberse al insuficiente conocimiento y/o experiencia, por parte de los estudiantes, de las normas que subyacen al uso lingüístico adecuado en situaciones comunicativas auténticas de los miembros de esa comunidad.

Esta investigación y por tanto sus resultados tienen varias limitaciones, como el número de sujetos que se han podido obtener y las limitaciones impuestas por la propia prueba, intrínsecas a las pruebas de pragmática. Sería necesario realizar otros estudios representativos que confirmaran estos resultados. 
Siendo que la pragmática de una lengua es probablemente el componente más difícil de adquirir en una LE (Wyner, 2018), las implicaciones didácticas de esta investigación van encaminadas necesariamente a reforzar significativamente la enseñanza de la pragmática en el aula de FLE. Para ello, sería necesario crear e implementar instrumentos pedagógicos pragmáticos ad hoc para la clase de lengua, y previamente sensibilizar a los profesores a la importancia y necesidad de la pragmática en la enseñanza del FLE.

\section{Referencias bibliográficas}

Alcón, Eva \& María Pilar SAFont. 2008. "Pragmatic awareness in second language acquisition" in CENOz, Jasone \& Nancy H. Hornberger (eds.). Encyclopedia of language and education. Berlin, Springer, vol. 6, 193-204.

Ariel, Mira. 2010. Defining pragmatics. Cambridge, Cambridge University Press.

Bachman, Lye F. 1990. Fundamental considerations in language testing. Oxford, Oxford University Press.

BARDière, Yves. 2016. "De la pragmatique à la compétence pragmatique. À la recherche d'indices dans le CECRL" in Recherches en didactique des langues et cultures. Les Cahiers de L'Acedle, $\mathrm{n}^{\mathrm{o}}$ 13-1 <http://journals.openedition.org/rdlc/462> [22/05/2020].

Bardovi-Harlig Kathleen \& Zoltan DörnyeI. 1998. "Do language learners recognize pragmatic violations? Pragmatic versus grammatical awareness in instructed L2 learning" in TESOL Quarterly, n 32(2), 233-262.

Bardovi-Harlig, Kathleen. 2009. "Conventional expressions as a pragmalinguistic resource: Recognition and production of conventional expressions in L2 pragmatics" in Language Learning, $\mathrm{n}^{\mathrm{o}}$ 59, 755-795.

Bella, Spyridoula. 2012. "Pragmatic awareness in a second language setting: The case of L2 learners of Greek" in Multilingua, $\mathrm{n}^{\mathrm{o}} 31,1-33$.

Blum-KulKa, Shoshana, Juliane HOUSE \& Gabriele KaSPer (eds.).1989. Cross-cultural pragmatics: Requests and Apologies. Norwood, N.J. Ablex.

Brown, Penelope \& Stephen C. Levinson. 1987. Politeness: Some Universals in Language Use. Cambridge, Cambridge University Press.

CAnale, Michael. 1983. "From communicative competence to communicative language pedagogy" in RichaRds, Jack C. \& Richard Schmidt (eds.) in Language and communication. London, London Group Ltd, 2-27.

Canale, Michael \& Merrill Swain. 1980. "Theoretical Bases of Communicative Approaches to Second Language Teaching and Testing" in Applied Linguistics, $\mathrm{n}^{\mathrm{o}}$ 1(1), 1-47.

Celce-Murcia, Marianne, Zoltan Dornyei \& Sarah Thurrell. 1995. "Communicative competence: A pedagogically motivated model with content specifications" in Issues in Applied Linguistics, $\mathrm{n}^{\circ} 6$ (2), 5-35. 
CoHen, Andrew D. 2010. "Coming to terms with pragmatics" in IshiHaRA, Noriko \& Andrew CoHEN. Teaching and learning pragmatics: where language and culture meet. Harlow, Pearson Education, 3-20.

Cohen, Andrew D. 2018a. “An Introduction to Pragmatics for Learners and Teachers" in Cohen, Andrew D. Learning Pragmatics from Native and Nonnative Language Teachers. Bristol, Blue Ridge Summit, Multilingual Matters, 1-25.

Cohen, Andrew D. 2018b. "Ideas for Teaching Pragmatics and for Motivating Learners" in Cohen, Andrew D. Learning Pragmatics from Native and Nonnative Language Teachers. Bristol, Blue Ridge Summit, Multilingual Matters, 211-240.

Cohen, Andrew D. 2018c. "The Assessment of Pragmatics" in CoHen, Andrew D. Learning Pragmatics from Native and Nonnative Language Teachers. Bristol, Blue Ridge Summit, Multilingual Matters, 125-153.

Consejo de Europa. 2002. Marco común europeo de referencia para las lenguas. Aprender, enseñar, evaluar. Madrid, Instituto Cervantes.

Conseil De L'Europe. 2018. Cadre européen commun de référence pour les langues. Apprendre, enseigner, évaluer. Volume complémentaire avec de nouveaux descripteurs. $<$ https://rm.coe.int/cecr-volume-complementaire-avec-de-nouveauxdescripteurs/1680787 $5 \mathrm{~d} 5>[12 / 05 / 2020]$.

Cook, Misty \& Anthony LiddicoAT. 2002. "The development of comprehension in interlanguage pragmatics: The case of request strategies in English" in Australian Review of Applied Linguistics, $\mathrm{n}^{\circ} 25,19-39$.

Dalmau, María Sabaté \& Hortensia Curell Gotor. 2007. "From "sorry very much" to "I'm ever so sorry": Acquisitional patterns in L2 apologies by Catalan learners of English" in Intercultural Pragmatics, $\mathrm{n}^{\circ} 4$ (2), 287-315.

Dumitrescu, Domnita \& Patricia Lorena Andueza. 2018. L2 Spanish Pragmatics. From Research to Teaching. London, New York, Routledge.

ESLAMI-RAKESH, Zohreh. 2005. "Raising the pragmatic awareness of language learners" in ELT Journal, nº 59, 199-208.

GArcía, Paula. 2004a. "Pragmatic comprehension of high and low level language learners" in Teaching English as a Second or Foreign Language, $\mathrm{n}^{\mathrm{o}}$ 8, 1-10.

García, Paula. 2004b. "Developmental differences in speech act recognition: A pragmatic awareness study" in Language Awareness, $\mathrm{n}^{\circ}$ 13, 96-115.

GeYer, Naomi. 2007. "Self-qualification in L2 Japanese: An interface of pragmatics, grammatical, and discourse competences" in Language Learning n ${ }^{\circ}$ 57, 337-367.

Holttinen, Tuuli. 2017. "Passe-moi le sel" vs "Pourriez-vous me passer le sel, s'il vous plaît?". Le développement des stratégies de requête chez les apprenants finophones de FLE» in Granget, C., M.-A. Dat, D. Guedat-Bittighoffer et C. Cuet, Connaissances et Usages en L2, Knowledge and Usage in L2, SHS Web of Conferences, 38. <https:// www.shsconferences.org/articles/shsconf/abs/2017/06/shsconf_couls2016_00004/shsconf_ couls2016_00004.html> [04/04/2020] 
Hymes, Dell. 1972. “On communicative competence” in PRIDE, John B. \& Janet Holmes (eds.). Sociolinguistics. Harmonsworth, Penguin, 269-293.

ISHIHARA, Noriko. 2010a. "Collecting data reflecting the pragmatic use of language" in IsHIHARA, Noriko \& Andrew D. CoHEN. Teaching and learning pragmatics: where language and culture meet. Harlow, Pearson Education, 99-122.

ISHIHARA, Noriko. 2010b. "Theories of language acquisition and the teaching of pragmatics" in IshiHARA, Noriko \& Andrew D. CoHEN. Teaching and learning pragmatics: where language and culture meet. Harlow, Pearson Education, 99-122.

IsHIHARA, Noriko. 2010c. "Assessment of pragmatics in the classroom " in IsHiHARA, Noriko \& Andrew D. COHEN. Teaching and learning pragmatics: where language and culture meet. Harlow, Pearson Education, 99-122

IshiHara, Noriko \& Andrew CoHen. 2010. Teaching and learning pragmatics: where language and culture meet. Harlow, Pearson Education.

Kerbrat-Orecchioni, Catherine. 1992. Les interactions verbales (vol. II). Paris, A. Colin.

Kerbrat-Orecchioni, Catherine. 2005. "Politeness in France: How to Buy Bread Politely" in Hickey, Leo \& Miranda Stewart. Politeness in Europe, Clevedon, Buffalo, Toronto, Multilingual Matters, 29-44.

LeECH, Geoffrey N. 1983. Principles of pragmatics. London, Longman.

Martínez-Flor, Alicia \& Eva Alcón. 2007. "Developing pragmatic awareness of suggestions in the EFL classroom: A focus on instructional effects" in Canadian Journal of Applied Linguistics/Revue canadienne de linguistique appliquée, $\mathrm{n}^{\circ} 10$ (1), 47-76.

Martins, Cidalia \& Jean-Jacques Mabilat. 2016. Guide de communication en français. Paris, Didier.

Niezgoda, Kimberly \& Carsten Roever. 2001. "Pragmatic and grammatical awareness: A function of learning environment?" in Rose, Kenneth \& Gabriele KaSPER (eds.). Pragmatics in language teaching. New York, Cambridge University Press, 63-79.

PeARSON, Lynn. 2018. "L2 Spanish pragmatics instruction at the novice level: Creating meaningful contexts for the acquisition of grammatical forms" in DumitresCU, Domnita \& Patricia Lorena Andueza. L2 Spanish Pragmatics. From Research to Teaching. London, New York, Routledge, 214-231.

Rafieyan, Vahid. 2014. "Effect of Pragmatic Awareness on Comprehension and Production of Conventional Expressions" in Theory and Practice in Language Studies, vol. 4, n 7 , 1352-1358.

Ruiz De Zarobe, Leyre. 2014. "Pour une introduction de la pragmatique dans l'enseignement du français en Espagne" in Synergies Espagne, n 7, 133-143.

Ruiz De Zarobe, Leyre. 2019. "Enseñar la competencia pragmática” in RuIZ De ZARobe, Leyre \& Yolanda RUIz De Zarobe. Enseñar hoy una lengua extranjera. Barcelona, Octaedro, 140-183. <https://octaedro.com/wp-content/descargas/30809-Ense\%C3\%B1ar-hoyuna-lengua-extranjera.pdf $>$ [03/04/2020]. 
SCHAUER, Gila. 2006. "Pragmatic awareness in ESL and EFL contexts: Contrast and development" in Language Learning, $\mathrm{n}^{\circ}$ 56, 269-318.

SchmidT, Richard W. 1993. "Consciousness, learning and interlanguage pragmatics", in KASPER, Gabriele \& Soshana Blum-KulKA in Interlanguage pragmatics. Oxford, Oxford University Press, 21-42.

Schmidt, Richard W. 2001. "Attention" in Robinson, Peter (ed.). Cognition and second language instruction. Cambridge, Cambridge University Press, 3-32.

Taguchi, Naoko. 2009. Pragmatic competence. Berlin, New York, Mouton de Gruyter.

TAGUCHI, Naoko. 2015. "Instructed pragmatics at a glance: Where instructional studies were, are and should be going" in Language Teaching, no 48 (1), 1-50.

TAKAHASHI, Satomi. 2015. "The effects of learner profiles on pragmalinguistic awareness and learning" in System, $\mathrm{n}^{\circ}$ 48, 48-61.

Thomas, Jenny. 1983. “Cross-cultural pragmatic failure” in Applied Linguistics, $\mathrm{n}^{\circ}$ 4(2), 91 112.

WANARUK, Anchalee. 2008. "Pragmatic transfer in Thai EFL refusals" in RELC Journal, $\mathrm{n}^{\circ}$ 39(3), 318-337.

Wyner, Lauren. 2018. "The Development of Pragmatic Ability" in CoHEN, Andrew D. Learning Pragmatics from Native and Nonnative Language Teachers. Bristol, Blue Ridge Summit, Multilingual Matters, 26-56.

XIAO, Feng. 2015. "Proficiency effect on L2 pragmatic competence" in Studies in Second Language Learning and Teaching, $\mathrm{n}^{\mathrm{o}} 5$ (4), 557-581.

\section{Anexo}

\section{Prueba de comprensión}

A. Comment jugez-vous les énoncés suivants en italique?
a. Adéquat
b. Moins adéquat
c. Non adéquat

B. Pourquoi les jugez-vous ainsi?

1. À la fin d'une soirée où se sont réunis des invités, quelqu'un dit à l'hôtesse: -Merci beaucoup, Madame, c'était délicieux, ce plat en particulier: vous êtes un chef. 
2. Mme. Legrand accueille un conférencier très célèbre à l'aéroport. Le conférencier porte une valise très lourde. Mme. Legrand lui propose de l'aide. Elle dit: Je t'aide?

3. Dialogue:

Mme. Colbert: Bonjour. Donnez-moi un rosbif d'un kilo, s'il vous plaît.

Le Boucher: Il y en a un peu plus. Ça ira quand même?

Mme. Colbert: Oui

Le Boucher: Et quoi encore?

Mme. Colbert: Deux tranches de jambon, s'il vous plaît.

4. Yves propose à ses amis d'aller au cinéma ce soir. Il dit:

On se fait un ciné ce soir?

5. Vous recevez une lettre qui vous donne une bonne nouvelle. La lettre commence:

Monsieur, Madame,

J'ai le bonheur de vous informer que votre candidature a été retenue.

6. Dialogue

Un touriste: Excuse-moi, pour aller à la tour Eiffel, s'il te plaît?

Un passant: Vous êtes à pied?

Le touriste: Oui

Le passant: Alors, c'est très simple. Vous remontez les Champs-Elysées et au rond-point, vous prenez l'avenue Montaigne à gauche. Ensuite, vous allez tout droit jusqu'à la place de l'Alma. Vous tournez à gauche, quai Branly. Vous marchez 300 mètres et vous arrivez aux pieds de la tour Eiffel. Vous avez compris?

Le touriste: Oui. Merci beaucoup.

7. Au café

Alexandre: Excusez-moi. C'est libre?

Une cliente: Euh...non. J'attends quelqu'un.

8. Vous achetez un billet de train au guichet de la gare:

$J^{\prime}$ aimerais avoir un billet pour Milan.

9.Dans un vernissage.

Evelyne et Julie sont deux amies.

Evelyne: Qu'est-ce que c'est cette installation informe? Quelle horreur!

Julie: C'est le nouveau style de Jean-Yves. Tu n'aimes pas?

Evelyne: Je trouve ça nul. Dire qu'il peignait de si belles toiles, il y a quelques années!

Julie: Tu exagères. Disons que ça manque d'originalité!

10. Jean fait un compliment à Pierre sur son intelligence.

Pierre répond très sérieusement: Tu sais, je ne suis pas n'importe qui 


\section{Prueba de producción}

Vous êtes en France (en séjour d'études ou de travail) et vous vous trouvez dans les situations suivantes. Que dites-vous dans chaque cas?

1. Vous montez dans le bus et vous bousculez une dame âgée qui est sur le point de tomber par terre.

2. Vous devez rendre un travail pour le 30 mai, mais vous ne pouvez pas le finir pour cette date. Vous allez voir le professeur dans son bureau et vous lui demandez s'il est possible de rendre le travail quelques jours plus tard.

3. Une bonne copine à vous, Anne, vous demande de l'aide avec le travail de littérature qu'elle doit présenter en classe. Vous voudriez bien l'aider, mais vous avez un examen demain et vous refusez.

4. Vous aimez beaucoup la robe rouge que porte votre copine d'appartement. Vous lui faites un compliment.

5. Votre téléphone portable est tombé en panne et vous demandez à votre ami son téléphone portable pour faire un appel.

6. Vous invitez votre chez de bureau à votre anniversaire chez vous.

7. Vous avez beaucoup aimé la conférence sur Proust que vous venez d'entendre. À la fin de la conférence, vous vous approchez du conférencier et vous le complimentez pour la conférence.

8. Vous oubliez un rendez-vous que vous avez avec une amie pour lui expliquer un programme d'ordinateur. Une heure plus tard, vous vous en rendez compte, et vous lui téléphonez pour vous excuser.

9. Votre voisine, que vous ne connaissez pas trop, vous demande de garder son chien l'après-midi. Vous êtes occupé/e cet après-midi et vous refusez

10. Vous invitez à un ami français, Pierre, à dîner chez vous samedi. 